\title{
Mark Nichter: Global Health: Why Cultural Perceptions, Social Representations, and Biopolitics Matter
}

\author{
University of Arizona Press, 2008, 320pp (ISBN-10: 0816525749, \\ ISBN-13: 978-0816525744, paperback)
}

\section{Susan M. Glover}

Published online: 23 May 2009

(C) The Author(s) 2009. This article is published with open access at Springerlink.com

Traditionally public health initiatives have focused on questionnaires and short interviews to identify the depth of local knowledge about a particular disease or to identify cultural barriers to health programs. Global Health by Mark Nichter (2008) demonstrates how qualitative in-depth ethnographic studies enhance our understanding of the relationship between local beliefs, rhetoric, and public health. This book challenges social scientists and medical practitioners to go beyond simplistic "cultural barrier" explanations for the failure of particular public health projects in the developing world. Health practitioners, patients, and social scientists all have their own culturally relevant strategies that determine if medicine is practiced in such a way as to effectively aid local health.

The first section of the book is concerned with how local perceptions of the classification, causes and treatment of infectious disease shape responses to public health endeavors. This section is packed with cautionary tales of the complexity of public health programs in the developing world. Nichter organizes these examples for the reader into themes about local understanding of the body, health, diet, risk, illness and pharmaceuticals. For example, local understanding of what is considered healthy can shape who seeks medical care. In Cameroon, Ghana, and Nigeria blood in the urine of both male and female teens is considered part of normal development. This belief leads to delays in the treatment of schistosomiasis. This first section of the book strongly demonstrates that a detailed understanding folk epidemiology is crucial for constructing effective health plans.

S. M. Glover $(\bowtie)$

University of California,

Davis, CA, USA

e-mail: smglover@ucdavis.edu
Nichter follows with a discussion of the interaction between public health rhetoric and local interpretations of those messages. Messages may hybridize when multiple public health messages are received. In northeastern Thailand public health messages about diarrhea, which encouraged people to keep water uncontaminated, were crossed with messages to control mosquito populations by covering water storage jars. Villagers thought that mosquito eggs in drinking water made people sick, and that covering jars would reduce diarrhea. Public health initiatives also interact with traditional medicine. For instance, side effects of drugs may be valued because they demonstrate that the medicine is effective and powerful. Thus, side effects are highly valued in areas where many counterfeits are found on the market, such as West Africa.

However, patients are not the only players in the public health system. Nichter also investigates how rhetoric mobilizes popular support. Local perception of the government shapes the enthusiasm with which public health initiatives are followed. In the Dominican Republic, community members understood the importance of controlling the mosquito population to prevent dengue fever. However, the locals did not cooperate because the reputation of the government was so poor that all of its programs were suspect. In fact, politicians have large incentives to make highly visible and capital-intensive public health demonstrations to increase popularity, but this may make little improvement on local health. Rhetoric may also falsely represent the best way to implement medicine by presenting ideals rather than reality. For example, health programs aimed at young mothers may not be successful if these women do not have power within the household to make decisions about purchases or to leave the house unaccompanied.

The last section of the book examines the possibilities for the next generation of global health research. He 
stresses the need to see "tropical diseases" as diseases of poverty and not geographical happenstance. Naturalizing these diseases draws attention away from the social and political dimensions of their spread. He also calls for a decreased emphasis on national boundaries for both the sources of public health funding as well as treatment. This poses serious coordination challenges in areas with many nation-states as well as decentralized governments. However, these challenges must be overcome for both pure humanitarian reasons as well as self-interest for the health of citizens in developed nations. A social science perspective, which views disease interacting in a larger cultural, historical, geographical, and biological framework, has potential for contributing to the challenges that global health presents.

Nichter provides convincing evidence that a deeper understanding of local culture is crucial for improving medical care. The sheer number of case studies convinces me that the complex relationships between public health rhetoric, local understanding, and local medical practice are unlikely to be fully understood using only the applications of quick surveys or questionnaires. Qualitative in-depth ethnographies have the potential to increase the likelihood that public health programs will succeed.

While this book presents an excellent review of medicine in the developing world, I believe a coherent science of global health has yet to be fully developed. The long list of case studies is organized by themes, which allows the reader to identify factors that are frequent challenges as well as construct a sort of global health taxonomy. However, the findings of each case study only seem relevant to particular unique cultural circumstances. A more cohesive theoretical perspective is necessary for looking at larger patterns of human behavior. For instance, how practical are folk perceptions of disease? Even if they are biologically incorrect, do they lead individuals to make good decisions? Also, when do we expect the most tension between Western medicine and traditional medicine? When do we expect the least? What lessons can we learn about public health in the developed world from identifying the importance of folk epidemiology in developing nations? Do patterns of program failure indicate something universal about human psychology?

I agree with Nichter that social science theory has great potential to advance global health. This is where I would expect a more cohesive theoretical perspective to start to develop. As he suggests, researching disease as part of a larger system (other diseases, politics, economics, religion, life-history, geography, etc.) has the ability to advance the science of global health. However, I would say that the social sciences could contribute more than Nichter suggests. Social scientists have long been interested in understanding the forces that create or destroy communities, social inequality, power, the importance of religion and ritual for dealing with environmental challenges, and the relationship between humans and their environments, all of which are relevant to global health research. I believe that as global health develops the research connections to social sciences will only become stronger.

Overall, this book provides an excellent review of the last few decades of international health research as well as suggestions for where future research should lead. It paints an accurate picture of the current challenges posed to both social scientists and field practitioners for improving public health in the developing world as well as encouraging the field to develop in productive ways.

Open Access This article is distributed under the terms of the Creative Commons Attribution Noncommercial License which permits any noncommercial use, distribution, and reproduction in any medium, provided the original author(s) and source are credited. 